\title{
The Logic of Relative Frustration: Boudon's Competition Model and Experimental Evidence
}

\section{Joël Berger* and Andreas Diekmann}

\author{
ETH Zurich, Clausiusstrasse 50, 8092 Zurich, Switzerland \\ *Corresponding author. Email: joel.berger@soz.gess.ethz.ch \\ Submitted March 2014; revised May 2015; accepted June 2015
}

\begin{abstract}
An improvement in the availability of opportunities for actors in a social system (e.g. a society or a firm) can coincide with a growing rate of frustrated individuals. For instance, uprisings have repeatedly been preceded by forms of political liberalization that have provided greater opportunities (the so-called Tocqueville paradox). In organizations, satisfaction with regard to promotion opportunities can be negatively associated with objective chances of promotion. Raymond Boudon has proposed a game-theoretic competition model, which specifies the micro-mechanisms that produce these puzzling phenomena at the aggregate level and clarifies the conditions under which they emerge. We conducted three laboratory experiments to test the model's predictions, making our study the first empirical test of Boudon's model. The results are mixed: when opportunities increased, the rate of the relatively frustrated losers in the group remained constant, or increased only slightly. However, when applying another aggregation rule, which accounts for all social comparison processes and does not merely focus on the losers, an increase in relative frustration under improved conditions was observed. Our results imply that under specific conditions, there is a trade-off between opportunities and social mobility, on the one hand, and social inequality and relative frustration, on the other.
\end{abstract}

\section{Introduction}

Surprisingly, an improvement in a society's opportunities can coincide with there being a larger share of frustrated individuals. The first to discuss this puzzling phenomenon was Tocqueville, who claimed that the outbreak of the French Revolution was triggered by economic and social improvements (Tocqueville, 1952 [1856]). The factual truth of his historical account is not at issue here, but his thesis is of general interest in the social sciences. ${ }^{1}$ The phenomenon whereby political reforms or economic improvements can lead to an increase in the rate of frustrated individuals and, with a certain probability, to uprisings, is called Tocqueville's paradox (Neckel, 2010). Brinton (1965) and Coleman
(1990) discuss several historical examples of Tocqueville's paradox. A recent example is China, where rapid economic growth has coincided with growing dissatisfaction (Ishida, Kosaka, and Hamada, 2014); as a consequence, Tocqueville's oeuvre has become popular among the party elite (Pei, 2013).

Related to the Tocqueville paradox is Durkheim's observation of rising suicide rates during rapid economic growth (Durkheim, 1952 [1897]). The effect of more overall frustration under improved circumstances does not only appear at the societal level, it also appears within organizations. In their study of social mobility in the US army, Stouffer et al. (1965 [1949]) report the classic finding that satisfaction regarding promotion 
chances was lower in branches with high objective opportunities. Related, and no less interesting, examples are discussed by Gladwell (2013).

There are two widespread explanations for the phenomenon of greater aggregate frustration under improved conditions (Esser, 2001: 432-437). The first explanation assumes that frustration arises from intraindividual comparisons of an individual's expectations regarding a situation with the actual state of affairs. For instance, if, because of social improvements, expectations grow faster than actual improvement, frustration may arise (e.g. Durkheim, 1952 [1897]; Brinton, 1965). The second explanation suggests that frustration derives from inter-individual comparison. Stouffer et al. (1965 [1949]), as an example, proposed that soldiers evaluate their situation in relation to a reference group-in this case, promoted soldiers. A soldier who has not been promoted and finds himself among a majority of soldiers who have not been promoted, and only a small share of soldiers who have, feels less dissatisfied than does a soldier who has not been promoted but who finds himself among a group containing a large number of people who have been promoted (see also Pettigrew, 2015).

In the following discussion, when talking of frustration arising from comparison with either of the reference points (i.e. an individual's expectations or an individual's reference group), we use the term relative frustration. We use the terms intra-individual and inter-individual relative frustration when referring to either case separately. To avoid confusion, we will not use the term relative deprivation, which usually refers to frustration arising from inter-individual comparison (Runciman, 1966).

Based on verbally formulated relative frustration theory (e.g. Merton and Rossi, 1957), attempts to quantify the theory were made. Several authors have put forward the Gini coefficient, or a variant thereof, as a measure of inter-individual relative frustration at the aggregate level (e.g. Yitzhaki, 1979; Kakwani, 1984; see D’Ambrosio and Frick, 2007 for a literature review and an empirical application). Boudon (1982 [1977]: ch. 5$)^{2}$ introduced a game-theoretic model, which explains the puzzle of the existence of more frustration under improving (or better) conditions as the consequence of rational individual decisions in interdependent competition situations. ${ }^{3}$ As the opportunities in a social system improve, competition becomes fiercer, leading to more overall frustration. ${ }^{4}$ At a certain point, the direction of the correlation changes and more opportunities are accompanied by a decreasing frustration level. In short, the model predicts an inverse U-shaped path of aggregate relative frustration when opportunities increase. This, however, only happens under specific conditions. In other regions of the parameter space, a negative association between opportunities and relative frustration is possible.

While Boudon outlined his model in a rather sketchy manner, Raub (1984) formalized it rigorously and analysed it game-theoretically. Raub demonstrated that the core prediction of an inversely U-shaped rate of relative frustration holds under different behavioural assumptions, and he specified the boundary conditions of this phenomenon precisely. Kosaka (1986) relaxed the model assumption of homogeneous actors and found that social inequality between competitors dampens to some degree the effect of greater frustration under improving conditions. Yamaguchi (1998) demonstrated that this only holds true when contest mobility exceeds sponsored mobility (as defined by Turner, 1960), while the opposite is the case when sponsored mobility prevails. Manzo (2009) implemented the competition model as an agent-based simulation. With this different approach, he replicated his forerunners' findings that the effect of greater frustration under improved conditions only emerges in certain regions of the parameter space. Moreover, Manzo (2011) showed that network topology might impact both the spread and intensity of relative frustration. ${ }^{5}$ Specifically, social comparison in local networks, as compared with global comparison, fosters relative frustration.

While the competition model has been analysed and developed theoretically, to our knowledge there has never been an empirical test of the model's central implications. To fill this gap, we conducted three laboratory experiments that test the central hypothesis of an inversely U-shaped path of relative frustration under improving conditions.

\section{Boudon's Competition Model and Hypotheses}

\section{The Model}

We start with $N$ actors facing a decision regarding whether to invest resources, $C$, such as time, effort, or money, in a competition for a scarce and highly valued good, for instance a high-prestige position within a firm. There are $k$ prizes (e.g. positions) and $n$ investors (competitors). Because the good is scarce, it must hold that $k<N .^{6}$ While $k$ is common knowledge, the number of investors can range from 0 to $N$. Successful investors get access to the scarce good (e.g. promotion) and therefore receive the high pay-off $\alpha$, which is given by gross benefit $B$ (e.g. prestige, power, or money) minus investment costs $C$ (see Figure 1; see also Hedström, 2005: 57).

Because the probability of success depends on the total number of investors, the investment decision is strategic in nature. If the number of investors $n$ exceeds 


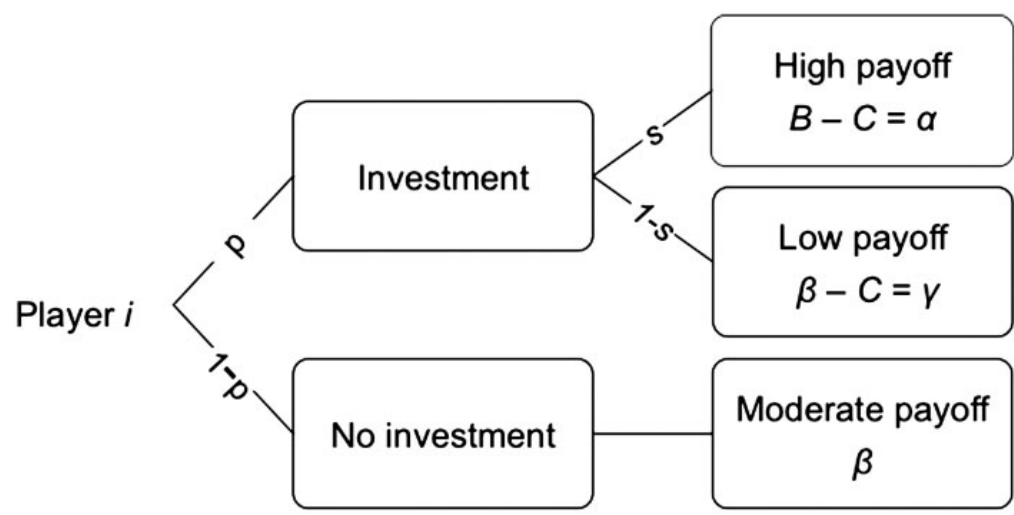

Figure 1. Individual decision situation: the strategy 'investment' is risky and leads to the high pay-off $\alpha$ with probability s and to the low pay-off $\gamma$ with probability 1 - s. The strategy 'no investment' leads to the medium pay-off $\beta$ with certainty.

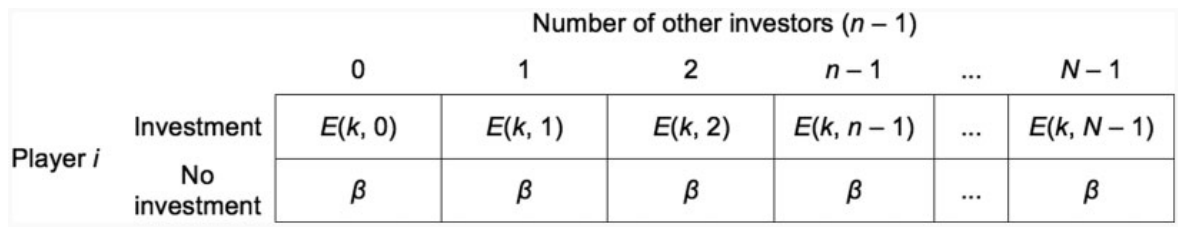

Figure 2. Game matrix from the perspective of player $i$. The expected pay-off $E(k, n)$ is determined by Equation (1).

the amount of free positions $k$, some investors necessarily fail to obtain a desired position. The losers are relatively frustrated because of both intra- and interindividual comparison: first, their expectation of receiving promotion as a result of investing is disappointed. Second, the losers adopt the successful investors as their reference group; while for the same stake $C$, the successful winners receive the high pay-off $\alpha$, the losers receive nothing in return. This is represented by the low pay-off $\gamma$. Sustainers (non-investors), e.g. individuals who decide not to expend time and money on training to qualify for promotion, will neither be promoted nor will they lose any resources. They consequently end up with the medium pay-off $\beta$. Formally, the pay-offs satisfy the inequalities $\alpha>\beta>\gamma^{7}$

Individual investment decisions depend on the opportunities provided by a given competition, which is determined by the number of scarce positions $k$ and by the cost-benefit ratio $Q$ :

$$
Q=\frac{B-\beta}{C} .
$$

$Q$ expresses how lucrative the winners' pay-off $\alpha$ is, in comparison with the sustainers' pay-off $\beta$, considering the investment costs $C$.

The probability of success $s$, for a player who chooses the strategy 'investment' is given by the ratio of the number of scarce positions $k$ to the number of investors $n$. This is provided that the number of investors exceeds the number of positions, and 1 otherwise:

$$
s=\left\{\begin{array}{lll}
\frac{k}{n} & \text { for } & k<n \\
1 & \text { for } & k \geq n
\end{array} .\right.
$$

Note that all $N$ players decide simultaneously whether to invest. Hence, before the decisions are made, the actual number of investors $n$ is unknown, while the number of positions $k$ is common knowledge. The higher the number of investors, the lower the chances of success for each player.

Given $\alpha, \gamma$, and $k$, the expected pay-off $E(k, n)$ for a specific number of investors $n$ is provided by:

$$
E(k, n)= \begin{cases}\frac{k}{n} \alpha+\frac{n-k}{n} \gamma & \text { for } k<n \\ \alpha & \text { for } k \geq n .\end{cases}
$$

With this information, the game matrix from the perspective of any player $i$ can be constructed (see Figure 2). In the matrix, the expected pay-off of the strategy 'investment' for each possible number of player $i$ 's competitors $(n-1)$ is depicted.

The model assumptions are in accordance with classical game theory. The rules of the game are common 


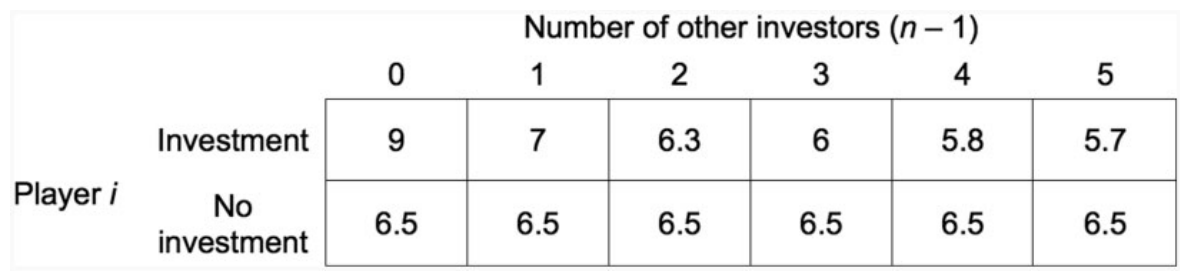

Figure 3. Game matrix from the perspective of player $i$. The expected pay-off $E(k, n)$ for the strategy 'investment', given the number of player i's competitors $(n-1)$, is determined by Equation (1). Parameter values: $k=1, \alpha=9, \gamma=5, N=6$. The pay-off $\beta$ for 'no investment' is fixed at 6.5 .

knowledge, and each player knows that all players are fully rational and maximize their expected utility.

So, what will a rational actor do in this situation? If the expected pay-off of 'investment' $E(k, n)$ exceeds the sustainers' pay-off $\beta$, independently of the actual number of investors, 'investment' is the dominant strategy, which implies that all players will invest. ${ }^{8}$ For the case that no dominant strategy exists, there is a threshold $n^{*}$ with the property that, as long as a maximum of $n$ " players choose to invest, the expectation of an investment exceeds the pay-off $\beta$. So there are $\left(\begin{array}{l}N \\ n *\end{array}\right)$ asymmetrical Nash equilibria ${ }^{9}$ in pure strategies, in which $n^{*}$ players choose 'investment', and $N-n^{*}$ players choose 'no investment'. Nevertheless, because homogeneous actors are assumed and communication is not possible, none of these equilibria is likely to be realized.

Rather, the rational solution lies in mixed strategies, with an optimal investment probability $p$. The mixed strategy solution of this game is in accordance with the rationality theory developed by Harsanyi and Selten (1988). According to the axioms of the Harsanyi-Selten theory, in a symmetric game, the solution must be a symmetric Nash equilibrium. The mixed-strategy equilibrium is the unique symmetric Nash equilibrium of the game and, therefore, it is the rational solution according to the Harsanyi-Selten theory.

Of course, there are other decision principles: a particularly simple one being the maximin strategy. Here, the maximin strategy is 'no investment', which yields pay-off $\beta$; the same pay-off a player expects when he uses the mixed equilibrium strategy. However, maximin is not an equilibrium strategy. If other players choose maximin, it is advantageous to deviate and to choose the strategy 'investment'.

Both the maximin strategy and the mixed equilibrium strategy give rise to an efficiency problem. In a cooperative game involving binding agreements, actors would agree on an asymmetric equilibrium with exactly $k$ investors, who are, for example, determined by the drawing of lots. Then, investors receive $\alpha$, and noninvestors receive $\beta$. This is the Pareto optimal pay-off vector with the welfare gain: $k \alpha+(N-k) \beta-N \beta>0$. However, without the possibility of agreeing on a binding contract, the Pareto optimal solution is not attainable.

The counter-intuitive phenomenon that additional positions lead to an increase in relative frustration occurs if a small increase in $k$ tempts an overly large number of players to choose to invest and, as a result, the increase in the number of losers exceeds, to a substantial degree, the number of additional positions. Roughly speaking, this is the case if the benefit of the strategy 'investment' is significantly greater than that of the strategy 'no investment', while the costs of investing are rather low; that is, if $Q$ is sufficiently high.

\section{Model Implications and Hypotheses}

We illustrate the mechanisms discussed using two numerical examples. Both of these examples have been implemented experimentally to test the model predictions.

\section{a) Mixed strategy case}

Let there be a social system of $N=6$ players choosing whether to compete for one of $k$ scarce positions. Sustainers receive a medium-level pay-off, $\beta=6.5$, with certainty. Successful investors receive the high pay-off $\alpha=9$, which is given by gross benefit $B=10.5$ minus investment costs $C=1.5$. Losers invest their resources in vain and end up with the low pay-off, in this case $\gamma=\beta-C=5$. The number of positions $k$ varies from 1 to 5 . In Figure 3, the game matrix for these parameter values and the case of 1 position is displayed. The payoff of the strategy 'no investment' is fixed at 6.5, while for a given player $i$, the expectation of 'investment' depends on the total number of her competitors $n-1$ and can be calculated using Equation (1). If player $i$ is the only investor, she will get 9 . Of course, the more players that enter the competition, the smaller is the expectation of the strategy 'investment' for any of the investors. Evidently, 'investment' is not a dominant strategy: as soon as two or more other players (i.e. in addition to $i$ ) 

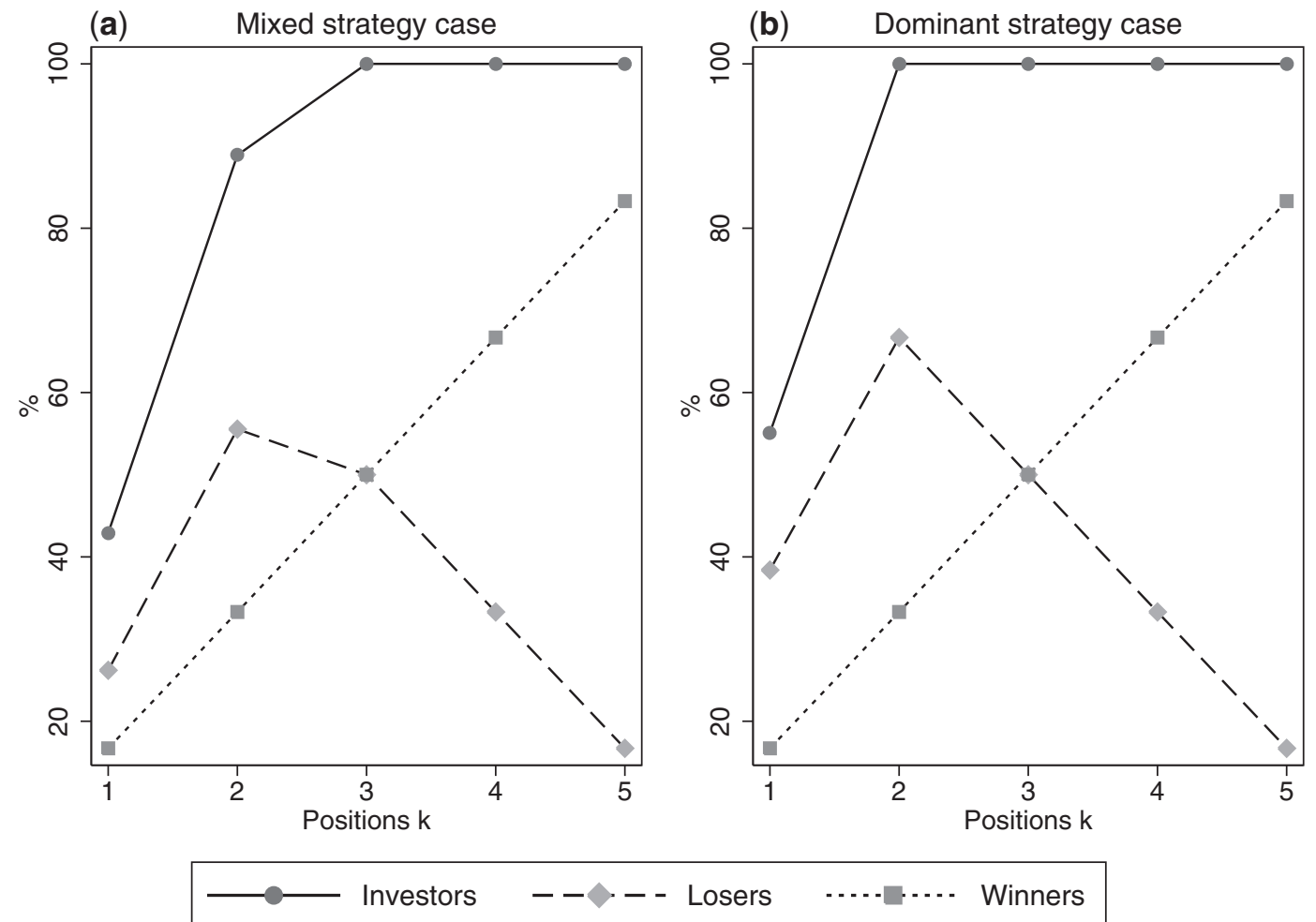

Figure 4. Model predictions: rates of investors, winners, and losers by number of positions $k$. (a) Mixed strategy case and (b) Dominant strategy case.

choose to invest, the expected pay-off of 'investment' is worse than the pay-off of 'no investment'. Hence, a rational actor will apply a mixed strategy.

The optimal mixture of both strategies can be gained by exploiting the principle of indifference. If all players choose to invest with the optimal probability $p^{*}$, all players are indifferent between their pure strategies. This results in a Nash equilibrium in mixed strategies (Nash, 1950). To derive $p^{*}$, the overall expected pay-off of 'investment' $E(k, \bullet)$, for a given value of $k$ and all possible permutations of investors, is equated with the pay-off of 'no investment' $\beta$ (see Equation (2)). Solving for $p$ yields the optimal investment probability $p$ " (Raub, 1984).

$$
E(k, \bullet)=\sum_{n=1}^{N}\left(\begin{array}{c}
N-1 \\
n-1
\end{array}\right) p^{n-1}(1-p)^{N-n} \cdot E(k, n)=\beta
$$

Given one position, for the parameter values specified above, the rational solution is to invest with a probability of $p^{*}=0.429$ and to sustain with $1-p^{*}=0.571$. The expected relative frequency of investors then equals $p *{ }^{10}$ Provided there are more investors than positions, the rate of winners in a social system of $N$ is given by the ratio of free positions to the total number of individuals $(k / N)$, and the loser rate is determined by subtracting the winner rate from the investor rate $\left(p^{*}-k / N\right)$. Should the share of investors undershoot the share of positions, every investor wins and there are no losers. Importantly, Boudon defines the rate of relative frustration in a social system as the loser rate, abstracting from the noninvestors.

For the parameter constellation discussed, the expected rates of investors, winners, and losers as a function of $k$ are plotted in Figure $4 \mathrm{a}$. If $k=1$, as derived from Equation (2), 42.9 per cent of all players are expected to invest. Because there are $(k / N) * 100=16.7$ per cent winners, there will be 42.9 per cent-16.7 per cent $=26.2$ per cent losers. When $k$ is doubled from one to two positions, the investor rate grows sharply, by 46.0 percentage points: the increase in positions leads to an overly large increase in investors and thus to fiercer competition. As a result, the rate of frustrated losers increases by 29.3 percentage points. This increase is sharper than the increase in the winner rate of 16.7 percentage points. Obviously, there are more additional losers than additional winners, even though the number of positions has doubled from one to two. 
If $k$ keeps rising, the loser rate starts decreasing: as soon as 100 per cent of the population is competing, additional positions can only diminish the rate of the relatively frustrated.

In short, the frustration rate follows an inversely Ushaped trajectory, with a maximum at $k=2$. We call the situation discussed the mixed strategy case because the paradoxical effect occurs at $k=2$, where an equilibrium in mixed strategies exists.

\section{b) Dominant strategy case}

In our second example, the winners' pay-off $\alpha$ is enhanced from 9 to 10 , such that 'investment' is the dominant strategy if there are two or more positions. In this case, we do not need to assume that actors use mixed strategies to deduce the hypothesis of an inversely U-shaped trajectory of relative frustration. We call this situation the dominant strategy case.

As depicted in Figure 4b, if there is only one prestigious position, 55.1 per cent of all players are predicted to invest, assuming players apply mixed strategies. Whichever decision rule actors follow in the case of one position, if there are two positions, 'investment' becomes the dominant strategy. This means that for every player, it is best to invest, independently of what the other players do. Even if all of player $i$ 's competitors choose to invest, $i$ 's expected gain from 'investment' is still greater than the pay-off of 'no investment'. Hence, all six players will compete for only two positions and, as a consequence, the level of frustrated losers reaches its maximum (100 per cent -33.3 per cent $=66.7$ per cent, compared with 38.4 per cent in the low-mobility system with one position). If $k$ keeps rising, more and more investors are promoted, while there are no additional losers. Again, the rate of relative frustration follows an inversely U-shaped functional form, with its maximum at $k=2$.

Importantly, the model highlights that an increase in relative frustration under improved chances of upward mobility does not necessarily occur. For instance, if 'investment' is the dominant strategy and all players invest anyway, additional positions $k$ coincide with fewer frustrated losers and more satisfied winners. Furthermore, if the expected pay-off of an investment is not too tempting, additional positions lead to a proportional investment rate, and relative frustration remains constant (see Supplementary Figure S1 in the online Supplementary Data (OSD)). Hence, the model implies that, at the aggregate level, there is no general law connecting opportunities to frustration rates. Depending on the parameter constellation, social improvements can lead to a higher as well as a lower frustration rate, or can leave it unchanged.
In our experiments, we chose parameters in a way that maximizes investment, and, thus, frustration when opportunities increase (for details, see OSD, Section 3), to test the main model prediction of an inversely Ushaped rate of relative frustration. More specifically, we aimed to test the following hypotheses:

H1: The higher the number of positions, the higher the number of investors according to the predictions of the game-theoretic model.

H2: The rate of the frustrated losers is an inversely $U$ shaped function of the number of positions, given the parameter values specified.

\section{Experimental Methods}

We conducted three laboratory experiments. In the first experiment, we implemented the parameters of the mixed-strategy case (the first of the two numerical examples discussed in the preceding session) in a withinsubjects design. ${ }^{11}$ Because the results deviated somewhat from the predictions (see below), we wanted to minimize the chance that the design was responsible for this. Specifically, giving feedback after each round of the experiment might have affected the subjects' investing behaviour. In Experiment 2, to exclude this we replicated the experiment in a between-subjects design. Additionally, we projected the pay-offs into the positive domain to eliminate loss aversion. The third experiment is a replica of the second case discussed in the preceding section-that is, the dominant strategy case. In this case, weaker rationality assumptions are necessary to derive the inversely U-shaped path of relative frustration from the model.

In Experiment 1, we compared competitions with one position (low-mobility condition), two positions (medium-mobility condition), and five positions (bigh-mobility condition) to test the hypothesis of an inversely U-shaped rate of relative frustration. In Experiments 2 and 3, we restricted ourselves to the comparison of low-mobility systems and mediummobility systems, and omitted high-mobility systems, so as to focus on the effect of an increase in the rate of relative frustration under improved conditions. An overview of all parameter values and predictions is given in Table 1.

Seventy-two students participated in Experiment 1, and 60 each in Experiments 2 and 3. In each experiment, subjects were randomly assigned to groups of six; separate competitions were held for each group. When all the individuals of a given group had decided whether to invest, $k$ winners were chosen randomly from among the 
Table 1. Parameter values and model predictions in Experiments 1, 2, and 3

\begin{tabular}{|c|c|c|c|c|c|c|c|}
\hline & \multicolumn{3}{|c|}{ Experiment 1} & \multicolumn{2}{|c|}{ Experiment 2} & \multicolumn{2}{|c|}{ Experiment 3} \\
\hline \multicolumn{8}{|l|}{ Parameters } \\
\hline Winners' pay-off $\alpha$ & 7 & & & 9 & & 10 & \\
\hline Sustainers' pay-off $\beta$ & 1 & & & 6.5 & & 6.5 & \\
\hline Losers' pay-off $\gamma$ & -3 & & & 5 & & 5 & \\
\hline Number of positions $k$ & $1,2,5$ & & & 1,2 & & 1,2 & \\
\hline Group size & 6 & & & 6 & & 6 & \\
\hline \multicolumn{8}{|l|}{ Predictions } \\
\hline & $k=1$ & $k=2$ & $\mathrm{k}=5$ & $k=1$ & $k=2$ & $k=1$ & $k=2$ \\
\hline Investors (per cent) & 39.7 & 83.3 & 100 & 42.9 & 88.9 & 55.1 & 100 \\
\hline Losers (per cent) & 23.0 & 50.0 & 16.7 & 26.2 & 55.5 & 38.4 & 66.7 \\
\hline
\end{tabular}

Note: Pay-offs in CHF.

investors of the corresponding group. In the first experiment, the participants played the competition game for six rounds, while only one round was played in Experiments 2 and $3 .^{12}$

\section{Relative Frustration in the Laboratory}

\section{Test of Hypotheses}

For all three experiments, we report descriptive statistics that are also visualized in Figure 5. Tests for statistical significance were conducted by means of logit models. The corresponding estimations are listed in Table A1 in the Appendix.

In Experiment 1, as expected, subjects invested with a higher probability as the number of positions was increased (Figure 5a). The investor rate rose from 36.1 per cent in the low-mobility condition $(k=1)$, to 54.9 per cent in the medium-mobility condition $(k=2)$, and reached 90.3 per cent in the high-mobility condition $(k=5)$. Both the investor rate of the low-mobility condition and the investor rate of the high-mobility condition differ significantly from the reference category $(k=2)$, at least at the 1 per cent significance level. Nevertheless, while predicted and observed values correspond quite well in the cases of one (39.7 per cent predicted, 36.1 per cent observed) and five positions (100 per cent predicted, 90.3 per cent observed), there were notably fewer investors than predicted in the medium-mobility system (83.3 per cent predicted, 54.9 per cent observed). Consequently, as depicted in Figure 5b, the rate of the relatively frustrated losers remained roughly constant when the number of positions was doubled from one position (20.8 per cent losers) to two positions ( 22.9 per cent losers, $P=0.748)$. Finally, and in line with the model, the loser rate of 10.4 per cent was lower in the high-mobility system in comparison with the medium-mobility system $(P=0.025)$. In short, no inversely
U-shaped rate of relative frustration was observed. Rather, the loser rate remained constant when the number of positions was doubled, and then decreased in the high-mobility condition.

In Experiment 2, in the low-mobility condition, 50.0 per cent of the subjects chose to invest, while 80.0 per cent entered the competition in the medium-mobility condition. This difference is statistically significant $(P=0.007)$, and the observed values approximate the predictions of 42.9 per cent $(k=1)$ and 88.9 per cent $(k=2)$ well (see Figure $5 \mathrm{c}$ ). Furthermore, in correspondence with the model, the loser rate of 46.7 per cent in the medium-mobility condition exceeds the loser rate in the low-mobility condition (33.3 per cent, see Figure $5 \mathrm{~d}$ ). However, because more losers than expected were generated in the low-mobility, and fewer in the medium-mobility condition, the difference in the loser rate amounts only to 13.3 percentage points, while the model predicts a 29.3-percentage point difference. Because the difference is not even half as large as predicted, it does not reach statistical significance.

In Experiment 3, 'investment' is the dominant strategy in the medium-mobility system, and therefore all six subjects of a given group were predicted to compete for only two positions. Despite this, as depicted in Figure 5e, only 73.3 per cent of all participants entered the competition. Because of this discrepancy, the loser rates did not differ much between the low-mobility condition (36.7 per cent, predicted: 38.4 per cent) and the high-mobility condition (40.0 per cent, predicted: 66.7 per cent, see Figure 5f, $P=0.731$ ).

To sum up, in all three experiments, investment increased with the number of positions, which means that Hypothesis 1 is supported from a qualitative point of view. Nevertheless, while investment behaviour corresponds neatly with the model at the extreme points of one and five positions, participants invested more 


\section{Experiment 1}

(a) Investors

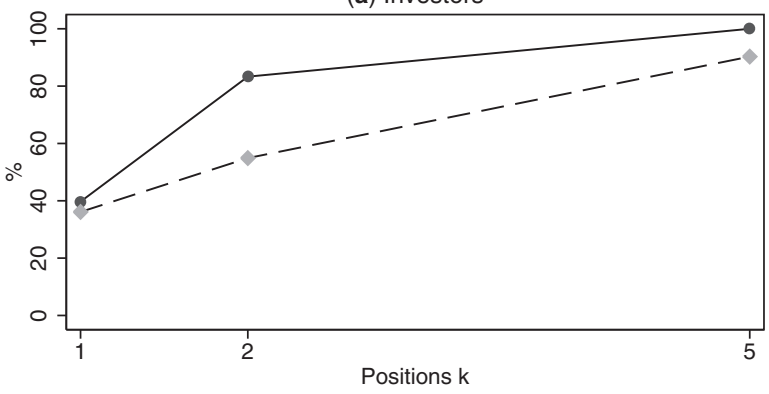

(b) Losers

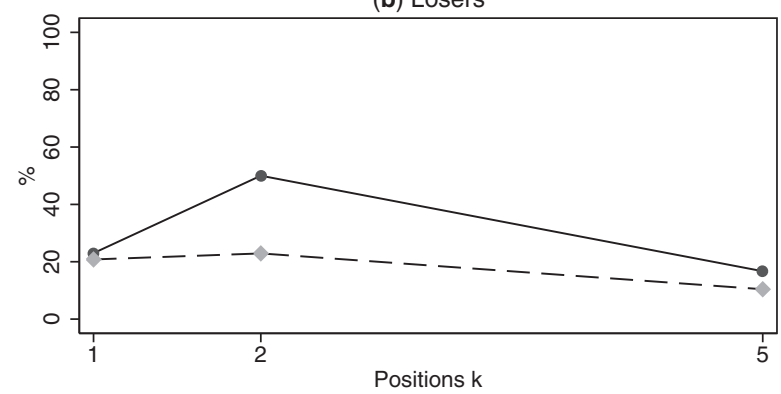

Experiment 2

(c) Investors

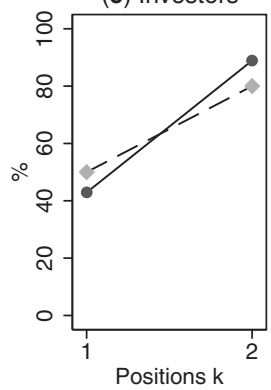

(d) Losers

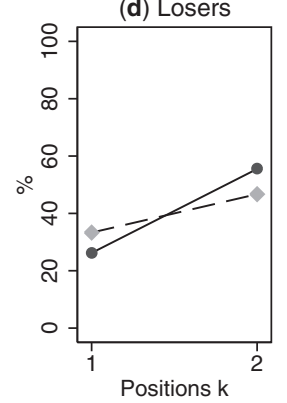

Experiment 3
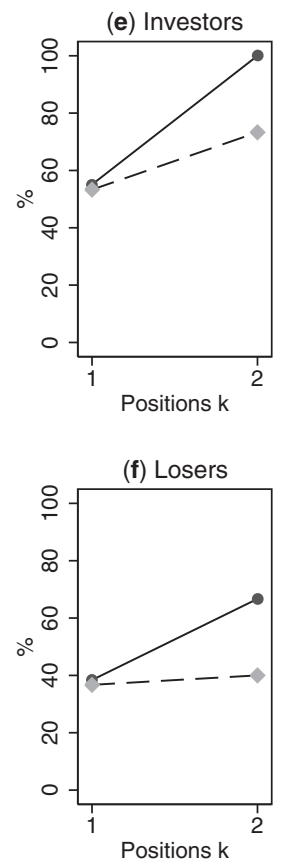

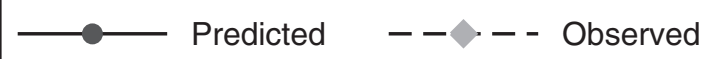

Figure 5. Predicted and observed rates of investors and losers by number of positions for all three experiments.

cautiously than expected in the medium-mobility condition with two positions. Consequently, frustration remained constant when the number of positions was doubled from one to two (Experiments 1 and 3 ) or increased only slightly (Experiment 2). Hypothesis 2 is thus rejected: the rate of the frustrated losers does not follow an inversely U-shaped trajectory but remains constant as positions are enhanced at a low level, and decreases in the high-mobility system-a result that lies between the model predictions and the naive view of decreasing frustration under improving conditions.

\section{Beyond the Loser Rate: Gini Coefficient}

Drawing on the literature on relative frustration discussed in the introduction, we questioned Boudon's narrow focus on the loser rate. While we agree with Boudon that the losers are the only ones being frustrated because of disappointed expectations, the sustainers might still be relatively frustrated because of comparisons with the winners. Boudon has acknowledged that even though the sustainers might not be as frustrated as the losers, they are also probably not as satisfied as the winners (Boudon, 1982 [1977]: 115). ${ }^{13}$
As a measure for relative frustration in a group, the Gini coefficient was suggested (Kakwani, 1984). The Gini accounts for all of the three types of actors (i.e. losers, winners, and sustainers) as well as for the different degrees of relative frustration perceived by losers and non-investors, by summing up the absolute differences in the pay-offs in a group. That is:

$$
\sum_{i=1}^{N} \sum_{j=1}^{N} d_{i j}
$$

where $d_{i j}$ is the absolute value of the difference in the pay-offs between actors $i$ and $j$. This summation procedure yields, after proper normalization, the desired measure. ${ }^{14}$

We computed the Gini coefficient predicted by the model and the corresponding empirical values for each experimental condition in Experiments 2 and 3 (see Figure 6 and Supplementary Figure S5, OSD). There are problems with the interpretation of the Gini coefficient in the presence of negative values, however. To resolve these problems, in Experiment 1, we calculated the variance as a substitute. ${ }^{15}$ 


\section{Variance and Gini coefficient}

(a) Experiment 1

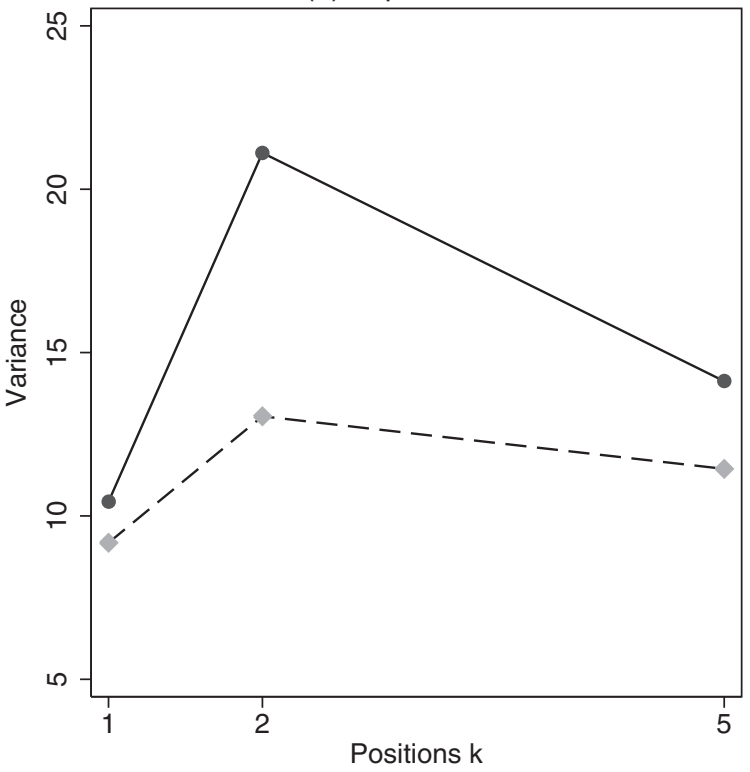

(b) Experiment 2

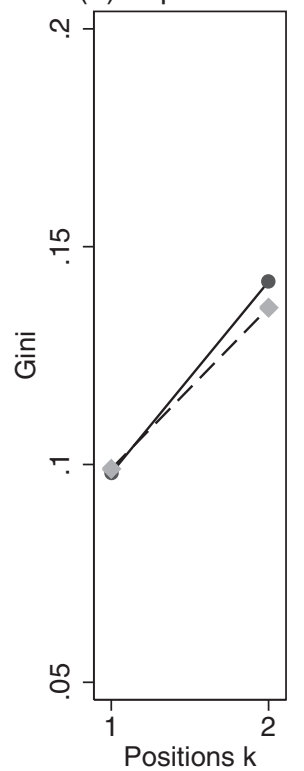

(c) Experiment 3

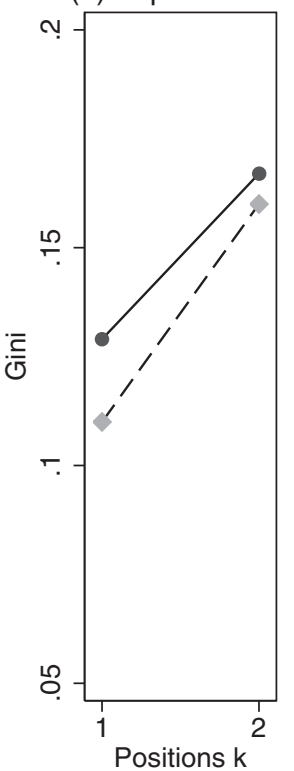

\section{$\longrightarrow$ Predicted $\longrightarrow---$ Observed}

Figure 6. Predicted and observed path of the variance (Experiment 1) and the Gini coefficient (Experiments 2 and 3 ).

Let us look at the predicted Gini coefficients and variances. In the beginning $(k=1)$, there are few winners and few losers, and the sustainers prevail-the group composition is largely equal. When positions increase sharply at a low level $(k=2)$, the formerly equal sustainers are divided into winners and losers. The dispersion in the pay-offs, and therefore the number of comparison processes, increases. Thereafter, the sustainers vanish, while the losers are diminished. Hence, after a peak, the Gini coefficient declines with increasing positions.

Not only the predicted, but also the empirical path of the Gini coefficient and the variance, are inversely Ushaped-at least in tendency. In Experiment 1, the variance increases significantly $(P=0.024)$, from 9.18 (predicted: 10.44 ) to 13.05 (predicted: 21.11), and finally decreases to 11.44 (predicted: 14.12), although not statistically significantly $(P=0.593) .{ }^{16}$ Note that even though the increase in the variance from the low-mobility to the medium-mobility condition is significant, this increase is not as steep as predicted. In Experiment 2, the Gini rises significantly $(P=0.006)$, from 0.106 (predicted: 0.098$)$ in the low-mobility condition to 0.140 (predicted: 0.142 ) in the medium-mobility condition. Similar results are observed in Experiment 3, where the Gini rises from 0.128 (predicted: 0.129$)$ to 0.161 (predicted: 0.167 ) when the positions are doubled $(P=0.025)$.

In all three experiments, the measures of dispersion increase when the number of positions is doubled and, in tendency, decrease thereafter. It is worth mentioning that this pattern is in line with the Kuznets curve. Kuznets (1955) argued that in the course of the economic growth that occurs during a society's transition from a rural to an industrial society, the trend of income inequality is inversely U-shaped.

Taken together, our findings suggest that Boudon's explanation of the puzzling phenomena reported by Tocqueville, Durkheim, and Stouffer et al. might need revision. While it is true that additional opportunities tempt an increasing share of actors to compete, in none of the three experiments was this increase as sharp as predicted. Consequently, relative frustration, defined as the loser rate, remained constant or increased only slightly when conditions improved at a low level. However, when not merely focusing on the losers, and accounting for all comparison processes between losers, sustainers, and winners, an increase in relative frustration is observed. 


\section{Discussion and Conclusions}

An improvement in the opportunities offered within a society (Tocqueville, 1952 [1856]) or an organization (Stouffer et al. 1965 [1949]) can lead to an increase in frustration at the aggregate level. Boudon (1982 [1977]) proposed a game-theoretic model to explain these counter-intuitive effects as the unintended consequence of strategic individual decisions. As in a social system, the chances of getting access to a scarce and lucrative good (e.g. a prestigious position within a firm) increase, then under specific conditions, the additional investors considerably outnumber the additional positions. As a consequence of this fierce competition, the rate of the relatively frustrated losers increases and Tocqueville's paradox emerges. When the number of positions is further enhanced, more and more investors achieve promotion and aggregate frustration diminishes again. In short, the model predicts an inversely U-shaped association between opportunities and relative frustration.

To test the main model predictions, we conducted three laboratory experiments with different parameter values-the first empirical test of Boudon's model, as far as we are aware. In accordance with the model, in all three experiments, participants invested with a higher probability, as the number of opportunities was enhanced. However, especially in the medium-mobility system, where we expected the rate of the frustrated losers to peak, participants invested more cautiously than predicted. As a consequence, the loser rate remained constant. When opportunities further improved, as expected, the loser rate decreased again. This result lies in between the model predictions of an inversely U-shaped rate of frustration and the intuitive belief in a decreasing frustration level under improving opportunities.

Post hoc, we questioned Boudon's conception of relative frustration, which narrowly focuses only on the losers of a competition. Following the received conception that relative frustration results from inter-individual comparison, we operationalized relative frustration as the Gini coefficient (Kakwani, 1984). In its essence, this measure sums over all of the differences in the players' pay-offs and, in doing so, captures all intra-group comparison processes. Summing over all differences is an alternative aggregation rule and, applying this rule, an inversely U-shaped rate of relative frustration is not only predicted by the model but is also found empirically. An increase in the Gini coefficient (or the variance, which we used as a substitute in Experiment 1) when chances improved was observed in all three experiments. This result is also in line with Kuznets' (1955) thesis of an inversely U-shaped rate of social inequality under improving economic conditions. Note, however, that we derived the inversely U-shaped path of the Gini coefficient from Boudon's model. Concerning the micromacro problem (Coleman, 1990), our modification demonstrates the importance of the question of how to transform individual decisions into macro-level effects. The implications of the model at the macro level crucially depend on the proper choice of aggregation rule.

Taken together, our experiments demonstrate that a more favourable opportunity structure can indeed generate more social inequality, perpetuating relative frustration. However, as derived from the model, this is only likely to occur under specific conditions (Raub, 1984; Manzo, 2009), e.g. when the expected benefit of investing in upward mobility is considerably larger than the benefit of not doing so, and when the increase in opportunities occurs at a low level.

Our study has some limitations. We tested model predictions with a specific set of parameter values and with (predominantly) Swiss students in a laboratory setting. It is possible that the predicted effect of an increasing loser rate would still occur when varying any of these features of the design. Further, we focused on whether investing behaviour changes with opportunities, as predicted by the model, and elicited frustration only by use of a verbal question. Introducing a behavioural measure of frustration would thus be a natural next step. Moreover, testing hypotheses derived from an expanded version of the model might be of interest. Given our results, mechanisms that reinforce frustration, such as comparison in local networks (Manzo, 2011) would be of special interest.

Finally, we would like to emphasize that Boudon's competition model is not restricted to the investigation of relative frustration. Rather, it provides a conceptual basis for theoretical and empirical investigations of the interconnection of competition structures, social mobility and inequality, status processes, and individual decision behaviour. The model generalizes 'winner-take-all markets' (Frank and Cook, 1995; Lutter, 2013) and is a fruitful expansion of classic market-entry models that have been previously used to describe situations such as these (e.g. Fischbacher and Thöni, 2008). It is worth mentioning that Boudon applied a variant of his model to analyse the effects of educational institutions on individual decisions (Boudon, 1979: 55-60; see also Raub, 1984: ch. 5). We believe that in the sociology of education, the competition model could be useful as an interdependent counterpart of parametric models (e.g. Breen and Goldthorpe, 1997). Given the actuality and sociological relevance of these phenomena, it seems worthwhile to further investigate the competition model and to exploit it as a theoretical framework for empirical research. 


\section{Notes}

1 Tocqueville's thesis of an improving economic situation before the French Revolution is controversial. Kruse (2005) states that there was an improvement in economic conditions in the 18th century in France but a period of stagnation in the 1780s (see also Hobsbawm, 1972 [1962]).

2 For reasons of simplicity, we use the most parsimonious version of the model, where actors decide between investing and not investing. In Boudon's notation: $B_{2}=C_{2}=0$ (Boudon, 1982 [1977]: 116-117).

3 Alternative explanations and models can be found in Davis (1959), Davies (1962), Elster (1991), and Gambetta (2005).

4 The model explains variation in relative frustration, with variation in the opportunities of a social system. Whether opportunities differ because of changes over time or are cross-sectional is irrelevant.

5 See also OSD, paragraph 4.2, on the intensity of relative frustration.

6 The two extreme cases are trivial: if $k=0$, there is no competition at all; 'no investment' is the dominant strategy. If $k=N$, every investor wins by definition and, again, there is no competition.

7 We tested the micro assumption that the losers are frustrated, i.e. less satisfied than the winners and the non-investors. The results suggest that this assumption holds. More specifically, the winners report the highest and the losers the lowest satisfaction, with the non-investors in between (see OSD).

8 For all of his co-players' strategy choices, a players' strictly dominant strategy leads to a higher pay-off than any other strategy available to the player (see e.g. Rasmusen, 2007).

9 A Nash equilibrium is a combination of strategies, in which no player has an incentive to unilaterally change his/her strategy.

10 For a detailed illustration of how to derive $p^{*}$, see OSD.

11 As is common in experiments with monetary incentives, we assume that, approximately, individuals' utility increases linearly with money in the pay-off range of the experiment.

12 See OSD for a detailed description of the experimental design and procedures.

13 See also endnote 4.

14 The Gini coefficient is defined as:

$$
\frac{1}{2 \bar{x}} \cdot \frac{1}{N^{2}} \sum_{i=1}^{N} \sum_{j=1}^{N} d_{i j},
$$

where $d_{i j}$ is the absolute value of the difference between person $i$ and $j$. The Gini is 0 when all payoffs are equal, and approximates 1 with increasing inequality. A meaningful interpretation of the Gini coefficient requires a ratio scale with a meaningful zero value, as with monetary pay-offs.

15 See Table A2 in the Appendix and OSD for further details of our analyses of Gini coefficients and the variance.

16 Here, we report results from the high-stakes condition only. In the low-stakes condition, the decrease in the variance from the high-to the low-stakes condition was more pronounced $(p=0.102)$. Note that splitting the sample into a high- and a low-stakes condition implies a decrease in statistical power.

\section{Acknowledgements}

We thank Norman Braun, Benita Combet, Ben Jann, Kunihiro Kimura, Anshul Kumar Sing, Matthias Näf, Thomas Voss, as well as Melinda Mills and three anonymous reviewers for helpful comments.

\section{Funding}

Funding provided by ETH Zurich, Chair of Sociology.

\section{Supplementary Data}

Supplementary data are available at ESR online.

\section{References}

Boudon, R. (1979). Generating models as a research strategy. In Merton, R. K., Coleman, J., and Rossi, A. S. (Eds). Qualitative and Quantitative Social Research. Papers in Honor of Paul F. Lazarsfeld. New York: The Free Press.

Boudon, R. (1982 [1977]). The Unintended Consequences of Social Action. London: Macmillan.

Breen, R. and Goldthorpe, J. H. (1997). Explaining educational differentials: towards a formal rational action theory. Rationality and Society, 9, 275-305.

Brinton, C. (1965). The Anatomy of Revolution. New York: Vintage Books.

Coleman, J. (1990). Foundations of Social Theory. Cambridge, MA: Harvard.

D'Ambrosio, C. and Frick, J. (2007). Income satisfaction and relative deprivation: an empirical link. Social Indicators Research, 81, 497-519.

Davies, C. (1962). Toward a theory of revolution. American Sociological Review, 27, 5-19.

Davis, J. A. (1959). A formal interpretation of the theory of relative deprivation. Sociometry, 22, 280-296.

Durkheim, E. (1952 [1897]). Suicide: A Study in Sociology. London: Routledge and Kegan Paul. 
Elster, J. (1991). Sour Grapes. Studies in the Subversion of Rationality. Cambridge: Cambridge University Press.

Esser, H. (2001). Soziologie. Spezielle Grundlagen. Band 6. Frankfurt a. M.: Campus.

Fischbacher, U. and Thöni, C. (2008). Excess entry in an experimental winner-take-all market. Journal of Economic Behavior and Organization, 67, 150-163.

Frank, R. H. and Cook, P. J. (1995). The Winner-Take-AllSociety. New York: Free Press.

Gambetta, D. (2005). Concatenations of Mechanisms. In Hedström, P. and Swedberg, R. (Eds.), Social Mechanisms. An Analytical Approach to Social Theory. Cambridge: Cambridge University Press, pp. 102-124.

Gladwell, M. (2013). David and Goliath. Underdogs, Misfits, and the Art of Battling Giants. New York: Hachette Book Group.

Harsanyi, J. C. and Selten, R. (1988). A General Theory of Equilibrium Selection in Games. Cambridge: MIT Press.

Hedström, P. (2005). Dissecting the Social. On the Principles of Analytical Sociology. Cambridge: Cambridge University Press.

Hobsbawm, E. J. (1972 [1962]). The Age of Revolution 17891848. London: Weidenfeld and Nicolson.

Ishida, A., Kosaka, K., and Hamada, H. (2014). A paradox of economic growth and relative deprivation. The Journal of Mathematical Sociology, 38, 269-284.

Kakwani, N. (1984). The relative deprivation curve and its implications. Journal of Business and Economic Statistics, 2, 384-395.

Kosaka, K. (1986). A Model of Relative Deprivation. Journal of Mathematical Sociology, 12, 35-48.

Kruse, W. (2005). Die Französische Revolution. Paderborn: UTB.

Kuznets, S. (1955). Economic growth and income inequality. The American Economic Review, 45, 1-28.

Lutter, M. (2013). Strukturen ungleichen Erfolgs. Winner-takeall-Konzentrationen und ihre sozialen Entstehungskontexte auf flexiblen Arbeitsmärkten. Kölner Zeitschrift für Soziologie und Sozialpsychologie, 65, 597-622.

Manzo, G. (2009). Boudon's model of relative deprivation revisited. In Cherkaoui, M. and Hamilton, P. (Eds.), Raymond Boudon-A Life in Sociology. Essays in Honour of Raymond Boudon. Oxford: Bardwell, pp. 91-121.

Manzo, G. (2011). Relative deprivation in silicio: agent-based models and causality in analytical sociology. In Demeulenaere,
P. (Ed.), Analytical Sociology and Social Mechanisms. Cambridge: Cambridge University Press, pp. 266-308.

Merton, R. K. and Rossi, A. S. (1957). Contributions to the theory of reference group behavior. In Merton, R. K. (Ed.), Social Theory and Social Structure. New York: The Free Press, pp. 225-280.

Nash, J. F. Jr. (1950). Equilibrium points in N-person games. Proceedings of the National Academy of Sciences of the United States of America, 36, 48-49.

Neckel, S. (2010). Verbesserungen führen zum Umsturz. Alexis de Tocqueville: "Der alte Staat und die Revolution" - das Tocqueville Paradox. In Neckel, S., Mijic, A., Scheve, C. and Titton, M. (Eds.), Sternstunden der Soziologie: Wegweisende Theoriemodelle des soziologischen Denkens. Frankfurt am Main: Campus, pp. 381-386.

Pei, M. (2013). The Tocqueville Paradox. The Indian Express, 11 January, posted online: <http://archive.indianexpress.com/ news/the-tocqueville-paradox/1057805/0> [accessed 24 January 2014].

Pettigrew, T. F. (2015). Samuel Stouffer and relative deprivation. Social Psychology Quarterly, 78, 7-24.

Rasmusen, E. (2007). Games and Information. An Introduction to Game Theory. Oxford: Blackwell.

Raub, W. (1984). Rationale Akteure, institutionelle Regelungen und Interdependenzen. Untersuchungen zu einer erklärenden Soziologie auf strukturell-individualistischer Grundlage. Frankfurt am Main: Peter Lang.

Runciman, W. G. (1966). Relative Deprivation and Social Justice. Berkley: University of California Press.

Stouffer, S. A., Suchman, E. A., de Vinney, L. C., Star, S. A. and Williams, R. M. Jr. (1965 [1949]). The American Soldier. Manhattan (Kansas): Military Affairs, Aerospace Historian Publishing.

Tocqueville, A. (1952 [1856]). L’Ancien Régime et la Révolution. Paris: Editions Gallimard.

Turner, R. H. (1960). Sponsored and contest mobility and the school system. American Sociological Review, 25, 855-862.

Yamaguchi, K. (1998). Rational-choice theories of anticipatory socialization and anticipatory non-socialization. Rationality and Society, 10, 163-199.

Yitzhaki, S. (1979). Relative deprivation and the Gini coefficient. The Quarterly Journal of Economics, 93, 321-324. 


\section{Appendix}

Table A1. Probability of investing and losing, respectively

\begin{tabular}{|c|c|c|c|c|c|c|}
\hline & \multicolumn{2}{|c|}{ Experiment 1} & \multicolumn{2}{|c|}{ Experiment 2} & \multicolumn{2}{|c|}{ Experiment 3} \\
\hline & (1a) Invest & (1b) Lose & (2a) Invest & (2b) Lose & (3a) Invest & (3b) Lose \\
\hline One position & $\begin{array}{l}-0.188 * * \\
(-2.76)\end{array}$ & $\begin{array}{l}-0.021 \\
(-0.32)\end{array}$ & Ref. & Ref. & Ref. & Ref. \\
\hline Two positions & Ref. & Ref. & $\begin{array}{l}0.300 * * \\
(3.05)\end{array}$ & $\begin{array}{l}0.133 \\
(1.29)\end{array}$ & $\begin{array}{l}0.200 * * \\
(2.41)\end{array}$ & $\begin{array}{l}0.033 \\
(0.34)\end{array}$ \\
\hline Five positions & $\begin{array}{l}0.354 * * * \\
(5.76)\end{array}$ & $\begin{array}{l}-0.125 * \\
(-2.24)\end{array}$ & - & - & - & - \\
\hline$N_{\text {groups }}$ & 12 & 12 & 10 & 10 & 10 & 10 \\
\hline$N_{\text {individuals }}$ & 72 & 72 & 60 & 60 & 60 & 60 \\
\hline$N_{\text {decisions }}$ & 432 & 432 & 60 & 60 & 60 & 60 \\
\hline$R^{2}$ & 0.18 & 0.03 & 0.08 & 0.02 & 0.12 & 0.08 \\
\hline
\end{tabular}

Note: Discrete change effects derived from logit models. Cluster-robust standard errors. $z$ statistics in parentheses. Controlled for session (not shown). $* P<0.05, * P<0.01, * * P<0.001$.

Table A2. Gini coefficients, variances, and results from statistical tests

\begin{tabular}{|c|c|c|c|c|c|}
\hline Experiment & $k$ & $G$ or $V$ & Standard error & Difference & Result \\
\hline \multirow[t]{3}{*}{1} & 1 & $V=9.18$ & 0.07 & $k=1-k=2$ & $\mathrm{z}=-2.25, P=0.024$ \\
\hline & 2 & $V=13.05$ & 0.078 & $k=5-k=2$ & $\mathrm{z}=-0.53, P=0.593$ \\
\hline & 5 & $V=11.44$ & & & \\
\hline \multirow[t]{2}{*}{2} & 1 & $G=0.106$ & 0.01 & $k=1-k=2$ & $t_{(d f=8)}=-3.19, P=0.006$ \\
\hline & 2 & $G=0.140$ & 0.003 & & \\
\hline \multirow[t]{2}{*}{3} & 1 & $G=0.128$ & 0.014 & $k=1-k=2$ & $t_{(d f=8)}=-2.30, P=0.025$ \\
\hline & 2 & $G=0.161$ & 0.003 & & \\
\hline
\end{tabular}

Note: G: Gini coefficient. V: Variance. Experiment 1: Cluster-robust standard error obtained by interval regression with parametric variance. Experiments 2 and 3: Unpaired $t$-tests. 